\title{
Population-Based Medical and Disease Management: An Evaluation of Cost and Quality
}

\author{
CHRISTOPHER G. WISE, Ph.D., M.H.A., ${ }^{1}$ VINITA BAHL, D.M.D., M.P.P., ${ }^{2}$ \\ RITA MITCHELL, ${ }^{2}$ BRADY T. WEST, M.A., ${ }^{3}$ and THOMAS CARLI, M.D. ${ }^{1}$
}

\begin{abstract}
Reports by the Institute of Medicine and the Health Care Financing Administration have emphasized that the integration of medical care delivery, evidence-based medicine, and chronic care disease management may play a significant role in improving the quality of care and reducing medical care costs. The specific aim of this project is to assess the impact of an integrated set of care coordination tools and chronic disease management interventions on utilization, cost, and quality of care for a population of beneficiaries who have complementary health coverage through a plan designed to apply proactive medical and disease management processes. The utilization of health care services by the study population was compared to another population from the same geographic service area and covered by a traditional feefor-service indemnity insurance plan that provided few medical or disease management services. Evaluation of the difference in utilization was based on the difference in the cost permember-per-month (PMPM) in a 1-year measurement period, after adjusting for differences in fee schedules, case-mix and healthcare benefit design. After adjustments for both case-mix and benefit differences, the study group is \$63 PMPM less costly than the comparison population for all members. Cost differences are largest in the 55-64 and 65 and above age groups. The study group is \$115 PMPM lower than the comparison population for the age category of 65 years and older, after adjustments for case-mix and benefits. Health Plan Employer and Data Information Set (HEDIS)-based quality outcomes are near the 90th percentile for most indications. The cost outcomes of a population served by proactive, population-based disease management and complex care management, compared to an unmanaged population, demonstrates the potential of coordinated medical and disease management programs. Further studies utilizing appropriate methodologies would be beneficial. (Disease Management 2006;9:44-55)
\end{abstract}

\section{INTRODUCTION}

$\mathbf{R}$ EPORTS BY THE Institute of Medicine and the Health Care Financing Administration have emphasized that the integration of med- ical care delivery, evidence-based medicine, and chronic care disease management may play a significant role in improving quality of care and reducing medical care costs. ${ }^{1,2}$ The specific aim of this project is to assess the im-

\footnotetext{
${ }^{1}$ UMHS Medical Management Center, University of Michigan, Ann Arbor, Michigan.

${ }^{2}$ Office of Clinic Affairs, University of Michigan, Ann Arbor, Michigan.

${ }^{3}$ Center for Statistical Consultation and Research, University of Michigan, Ann Arbor, Michigan.
} 
pact of an integrated set of care coordination tools and chronic disease management interventions on utilization, cost, and quality of care for a population of beneficiaries who have complementary health coverage through a plan designed to apply proactive medical and disease management processes.

The University of Michigan Health System's Medical Management Center (MMC) applies these tools and interventions in combination with a prospective health risk assessment, point-of-care information management, clinical decision support, multidisciplinary clinical oversight, and a clinical "Health Navigator" to deliver integrated health care to all patients in a defined population. Over the past 5 years, the MMC has provided these components in a unique commercial, self-funded health plan called "Partnership Health." Through Partnership Health, the MMC applies high-intensity care coordination and integration methods in a fee-for-service environment.

Our main hypothesis is that the MMC's application of these tools and interventions to the members in Partnership Health has resulted in improved quality of care at equal or reduced cost, as compared to usual care of a population enrolled in a traditional, unmanaged fee-forservice model.

\section{Significance}

The number of individuals with chronic disease is growing at an unprecedented rate due to the rapid aging of the population and the increased longevity of people with chronic disease. Unfortunately, there is ample evidence of deficiencies in both the processes and outcomes of care for persons with major chronic diseases. ${ }^{3-5}$ The structure of fee-for-service health care, organized around patient-initiated visits for episodic acute illness, has received much of the blame for this state of affairs. $3,6,7$

Disease management programs, collections of clinical and administrative resources targeted at improving care for specific diseases, represent a focused approach to overcoming the structural problems stemming from traditional methods of reimbursement and organization. Several studies have confirmed the effectiveness of disease management programs that employ evidence-based clinical protocols, encourage patient self-management, and provide clinical decision support for the care team. ${ }^{6-8}$ However, disease management programs that extend their boundaries to serve less severely ill patients or are implemented in multiple settings have shown no consistent effect on outcomes. ${ }^{9-13}$ The limited success of these broad-based disease management programs highlights the importance of integrating disease management into routine health care. Elderly patients with chronic disease are particularly disadvantaged by the lack of integration of disease management programs because they frequently have several coexisting chronic conditions. For many elderly persons, multiple comorbidities, rather than the presence of a single dominant chronic disease, is the major contributor to functional limitations ${ }^{14}$ and increased healthcare utilization. Enrollment in "competing" disease management programs for these people would greatly complicate efforts to coordinate care and likely impair the overall effectiveness of care.

Managed care organizations (MCOs) have responded to this problem by developing care management programs for members with multiple conditions, poor health status, and high utilization. Appropriate and continuous management of chronic disease in its early stages is key to preventing disability development ${ }^{14-16}$ and containing personal, social, and medical costs. ${ }^{17-19}$ However, most existing care management programs are either located within the MCO and focused on utilization management, or located within community-based social service agencies and focused on provision of personal care services. ${ }^{1}$ Neither type of program fosters appropriate links between care managers and ongoing medical care. ${ }^{19,20}$ This poor linkage is thought to be a major reason why care management has not generally been shown to affect healthcare utilization. ${ }^{15,19}$

Current efforts to deliver effective care for chronic illness focus on integrating "best practices" into routine fee-for-service and managed care settings. Chen et al consider disease management and care management to be complementary programs and identify comprehensive initial assessment, implementation and coordination, and reassessment and adjustment as the 
common elements in current best practices for each area. ${ }^{1}$ Any successful model for chronic illness care requires both disease management and care management elements, ${ }^{5}$ and integrates both elements in a "package" with tools that can be successfully implemented in a variety of practice settings, from primary care office to subspecialty clinic. ${ }^{5,21}$ The University of Michigan Health System's MMC has developed and pilot tested such a model over the past 5 years.

\section{METHODS}

\section{Medical and disease management}

The MMC is the University of Michigan Health System entity responsible for applying the medical management tools and interventions in an integrated healthcare delivery system for its defined populations. This encompasses interventions related to both complex medical management and disease management. Medical management activities are designed to identify the small number of members who account for a significant portion of total medical care costs. Provider-led disease management and complex care management programs then are available for referral of vulnerable, at-risk, and potentially high-cost patients with chronic disease.

The key personnel in the MMC responsible for applying the tools and administering the medical and disease management programs are as follows:

1. Coordinating Physician (CP), a primary care or specialist physician who assumes primary responsibility for the member's health care needs.

2. Health Navigator, a non-physician clinician care manager (ie, social worker, nurse) assigned to each member to assist in coordinating clinical care.

3. Clinical Oversight Team, a multidisciplinary group including physicians, non-physician clinicians, and key Health System leaders that meets weekly to help guide medical management.

4. Clinical Guideline Development Teams, composed of primary care and specialist clini- cians responsible for creation and maintenance of evidence-based clinical guidelines.

5. Provider-led Disease Management Programs, which supply comprehensive, evidencebased medical care for diabetes, congestive heart failure, asthma, coronary artery disease, and depression. The disease management programs range from educational and support services for patients with mild disease to multidisciplinary oversight with case management for those at the highest level of disease severity. All five MMC disease management programs have received Joint Commission on Accreditation of Healthcare Organizations (JCAHO) Disease Specific Care certification.

6. Clinical Information Data Support Systems, to provide data analytic and programming support for evidence-based medical and disease management, case finding, and provider feedback.

The core set of plan "tools" used by the MMC are as follows:

1. Health Risk Appraisal (HRA), designed to identify and prioritize individual members' health risks.

2. Health Summary, a summary of each member's medical care utilization (ie, physician visits, emergency room use, hospitalizations, major procedures, pharmacy utilization, ancillary services, health risks, and total costs), is provided to the CP quarterly.

3. Individual Health Care Plan (IHCP), completed by the $\mathrm{CP}$ with the patient and shared with the MMC. The IHCP serves as the basic summary of active health issues and is used to coordinate the care process as well as serve as a referral authorization.

4. Clinical Guidelines, evidence-based clinical guidelines covering the most common chronic and acute health problems, also serving as the core reference for outcome measurement protocols.

For the populations served, the MMC works closely with the Michigan Health Management Corporation, a University of Michigan owned subsidiary that provides third-party administrative services. This relationship assures that 
the MMC has timely and accurate data exchange of enrollment and claims data.

Enrolled members receive notification of the introduction of integrated care and availability of a Health Navigator by letter, but are not required to take any steps to "activate" medical management. The "content" of the intervention is thus transparent to the patient. The MMC process includes the following core elements:

1. Disease registry development. MMC staff assign patients to disease registries by collating information from the HRA, utilization data, personal interactions with the member, and identification by the CP (as designated on the IHCP).

2. Health Summary construction. For each patient, a Health Summary (including HRA results, prior utilization data, pharmacy fill data, and a list of active chronic diseases) is forwarded to his or her $\mathrm{CP}$, pending signature of appropriate confidentiality agreements. Health Summaries are updated quarterly and posted to the CP.

3. Active problem list creation and maintenance. During their first clinical encounters, patient and CP create an IHCP that is shared with the MMC and can be modified at the point of care during subsequent encounters.

4. Disease management services. All patients with confirmed diabetes, coronary artery disease, heart failure, asthma, and depression receive the baseline disease management package of educational services (for both $\mathrm{CP}$ and patient) and periodic outcome monitoring. Where appropriate, patients are referred for specialtylevel disease management and care management.

5. Clinical Oversight Team review. Patients who appear on multiple disease registries or who have complex medical problems trigger case review by the Clinical Oversight Team. The team reviews care options to determine the most effective ways to coordinate needed services, then feed this information back to the $\mathrm{CP}$ and care manager.

6. High risk notification. Emergency department encounters, hospital and nursing home admissions, and deaths are posted by fax within $24 \mathrm{~h}$ (or electronically in real time) to
CPs and the MMC Health Navigators to facilitate coordination of care.

7. Outcomes monitoring. Disease-specific outcome measures derived from clinical guidelines or selected by the guideline expert teams are assessed for all qualifying patients and reported on an aggregate level yearly.

8. Clinician feedback. CPs receive reports on all disease-based outcome measures (at both the individual patient and aggregate level) every 6-12 months. CPs also receive a quarterly Health Summary for each patient and have access to the IHCP. Ad hoc interactions between physicians, Health Navigators and the Clinical Oversight Team also provide critical feedback in more complex cases.

\section{Model for cost reduction and preliminary studies}

Reductions in cost can be achieved through improved impact of the tools and interventions on coordination and management, ${ }^{22}$ and a great deal of effort has gone into the design and assessment of the tools, interventions, and integrated medical management processes of the MMC. The Clinician Oversight Team has met weekly since 1998. Health Navigators have formalized their roles and performed an initial assessment of their impact on healthcare utilization, with preliminary evidence suggesting substantial salary cost offset through prevented emergency services and hospital admissions. ${ }^{23}$ The MMC evidence-based clinical guideline development process has been in place since $1995^{24}$; at present, the multidisciplinary guideline development teams also serve to design outcome measurement strategies for disease management. Discussions with the heart failure and asthma disease management programs identify significant improvement in diseasespecific outcome measures, quality of life, and healthcare utilization for enrollees in each program (written communication from William F. Bria, M.D., and Kim Eagle, M.D., May 2003).

As follow-up to the favorable preliminary results, a more thorough analysis was performed to evaluate the impact of medical and disease management provided by the MMC. The analysis compared the cost per-member-per-month (PMPM) of the population served by the MMC and a comparison population from the same 
geographic service area that was enrolled in an unmanaged fee-for-service health plan. The details of that evaluation are provided next.

\section{Comparison population}

The utilization of healthcare services by the population managed by the MMC was compared to another population which included active and retired employees from the same geographic service area. Unlike MMC members, the comparison population was covered by a traditional fee-for-service indemnity insurance plan that provided few medical or disease management services. Evaluation of the difference in utilization was based on the difference in the cost PMPM in a one-year measurement period, after adjusting for differences in fee schedules, case-mix, and healthcare benefits.

The outcomes reported in this study represent the activity over a consecutive 12-month time period from June 1, 2001 to May 31, 2002. During this study period, the MMC population consisted of 24,119 member months (2010 members), and the comparison group had 364,312 member months (30,360 members).

\section{Applying standardized fee schedule}

Standardizing provider fees eliminated the influence of different provider fee schedules on the relative costs PMPM between the two populations. Standardized fees were established for services such as acute care hospital days (general care and intensive care unit), physician services defined by CPT- 4 code, and drugs defined by the National Drug Code (NDC). The standardized fees were based on reimbursement rates for providers in southeast Michigan and therefore were realistic and not arbitrary. The standardized fee schedule was applied to claims for both populations. Therefore, the costs PMPM reported in this study are not the actual costs of paid claims. Instead, the results represent an unbiased and regionally representative comparison of the true relative costs between the MMC and the comparison population resulting from differences in the utilization of healthcare services.

The standardized fee schedule was applied to healthcare services for MMC and the comparison population and the costs for each mem- ber and for the two populations were summed. After identifying unique members and their member months of enrollment in the study year, unadjusted costs PMPM for each member and for the two populations were calculated. The volume and cost of facility-based imaging tests were estimated based on professional claims for the interpretation of imaging tests for both populations. The volume and cost of facility-based lab tests could not be estimated and were therefore excluded from the analysis.

\section{Case-mix adjustment}

To adjust the cost PMPM for differences in case-mix, the claims data for each population were grouped using Johns-Hopkins ACG CaseMix System ${ }^{\circledR}$. The ACG system is a respected instrument for case-mix adjustment and has been used in studies by many healthcare organizations and researchers during the past two decades. The software classified members of the MMC and comparison populations into 52 distinct ambulatory care groups that characterized their health status or illness burden. The cost PMPM was calculated for each of the 52 groups in each population. The approach to adjusting costs PMPM was to produce an estimate of the cost PMPM for an MMC population that had the same case-mix (ie, distribution of members among the 52 ACGs) as the comparison population. The adjustment was made to the MMC cost PMPM by applying the MMC cost PMPM for each ACG to the case-mix of the comparison population.

In this study, all medical, surgical, and behavioral health claims, with the exception of outpatient laboratory and radiology claims, were used for case-mix adjustment. The laboratory and radiology claims were excluded because they are known to be unreliable; these claims sometimes specify diagnoses to be "ruled out," rather than ones that were confirmed.

\section{Adjustment for benefit differences}

The MMC and comparison populations had different healthcare benefits. While both populations received the same outpatient drug benefits, the MMC group received benefits for medical/surgical care services, such 
as office visits and preventive care services, that the comparison population did not. A healthcare actuary evaluated the differences in benefits and determined that MMC had richer benefits for medical services that could produce approximately $9 \%$ higher costs than the comparison population. These estimates were applied to medical/surgical (not pharmacy) claims to adjust for differences in costs that could be attributed to differences in benefits. The medical/surgical claims cost PMPM for the comparison population increased by $9 \%$ after adjustments were made for differences in provider fees and population case-mix.

All outcomes are reported in both unadjusted and adjusted figures.

\section{RESULTS}

Figure 1 shows the age and gender distributions of the populations served by the MMC and the comparison population. The differences between the groups are striking. The comparison population has a notably larger distribution of members age 60 and above. This is because a majority of enrollees in the comparison population are retired employees and/or their spouses.

Figure 2 presents the distribution of members by the number of different health conditions. The MMC group has a higher percentage of members with four or less different health conditions; and nearly $15 \%$ of the comparison population have 10 or more conditions, com-

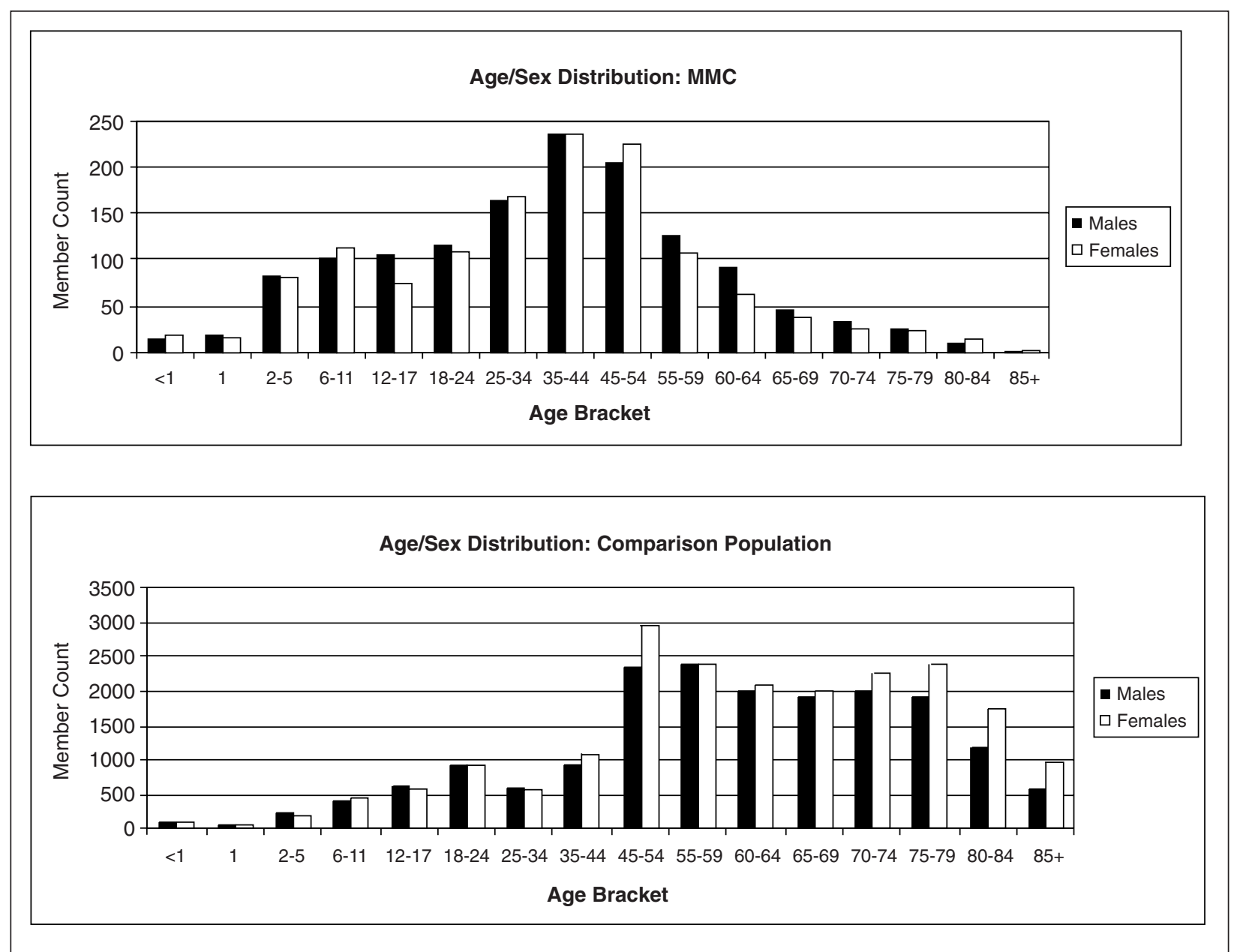

FIG. 1. Age and gender distribution of Comparison Population (CP) and Medical Management Center (MMC) population. 


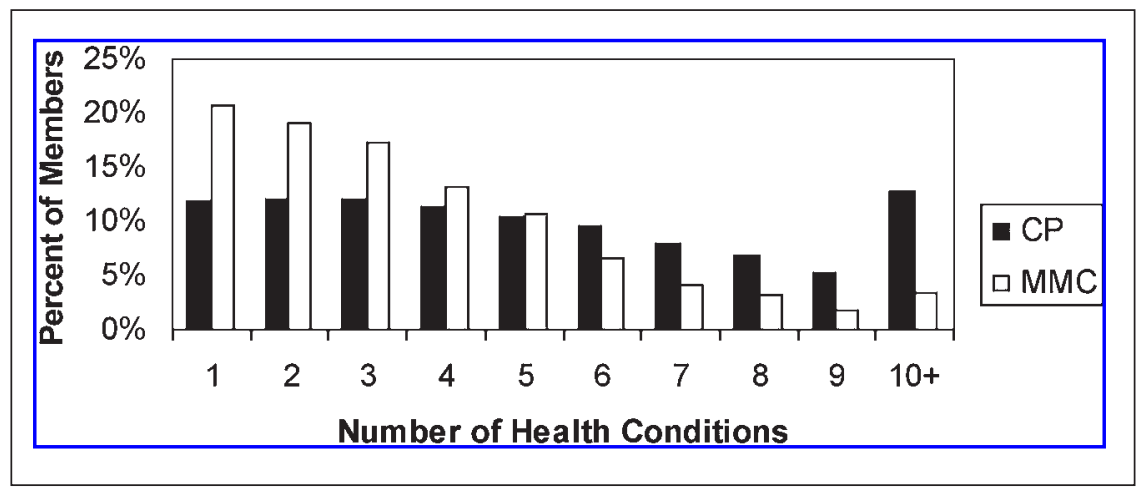

FIG. 2. Distribution of members by number of different health conditions of Comparison Population (CP) and Medical Management Center (MMC) population.

pared to less than 5\% of the MMC group. These findings are consistent with the older age distribution of the comparison populations because elderly individuals more commonly have a greater illness burden.

Figure 3 shows the most prevalent diseases for both the MMC and comparison populations. Hypertension, benign and unspecified neoplasm, disorders of lipid metabolism, and musculoskeletal signs and symptoms accounted for four of the top five most prevalent diseases in both groups. Depression was the sixth most prevalent in the MMC group but was not in the top 20 for the comparison population.

Figure 4 is a comparison of the cost PMPM between the MMC and comparison populations for the overall population and by age cat-

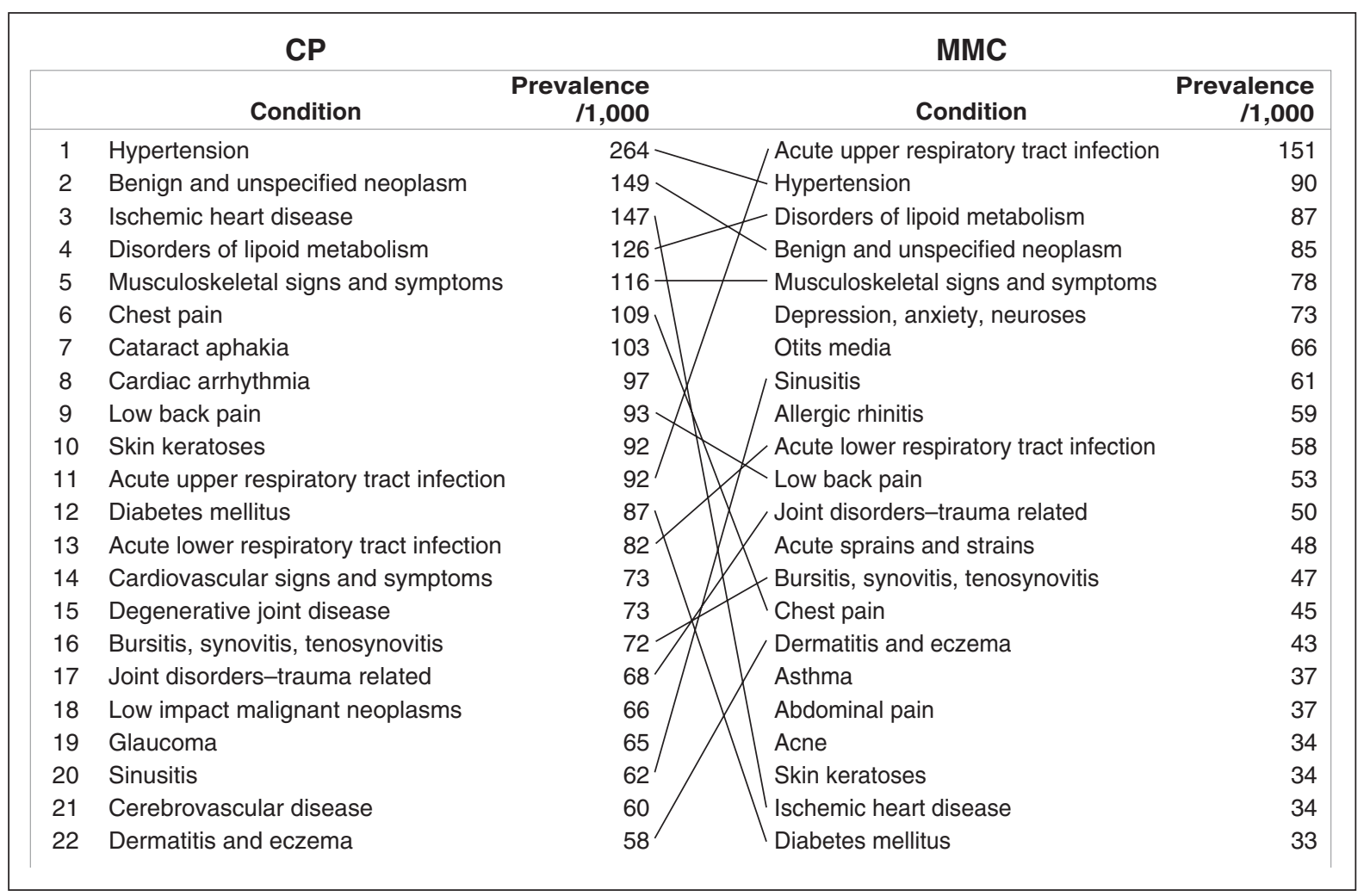

FIG. 3. Most prevalent diseases for Comparison Population (CP) and Medical Management Center (MMC) population. 


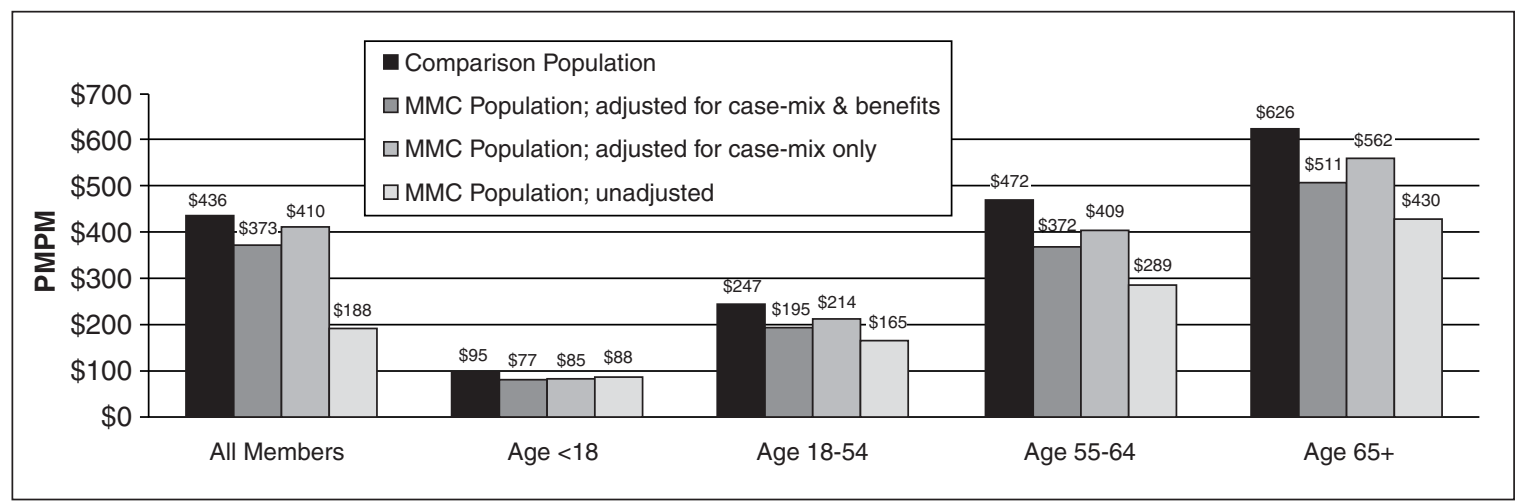

FIG. 4. Comparison of Per-Member-Per-Month (PMPM) costs by age category.

egory. For each age category, the left-most bar represents the costs of the comparison population. The bar directly to the right of the comparison population represents the MMC costs after adjustment for differences in both casemix and benefit plan. Next are the MMC costs after adjustment for case-mix only, followed by the unadjusted MMC costs. This representation is offered so the reader can observe the impact of the adjustments for differences in case-mix and benefits on costs.

The unadjusted costs PMPM for MMC are much less than the costs PMPM of the comparison population. For all members, the unadjusted cost PMPM for MMC was \$188 and the cost PMPM for the comparison population was $\$ 436$. Because of the substantial differences in the demographics and burden of illness between these two populations, comparisons of unadjusted figures are tenuous.

Case-mix adjustment produces a substantial increase in the costs PMPM of the MMC group, as expected. The comparison population was an older population with a greater burden of illness; case-mix adjustment is an attempt to estimate the cost of the MMC group if it was assigned a population with the same age and burden of illness distribution as that of the comparison population. Even after case-mix adjustment, the cost PMPM of the MMC group was \$26 PMPM less than that of the comparison population for all members and is also less in every age category.

Because the MMC group received benefits that were approximately $9 \%$ richer than the comparison population, adjusting for this dif- ference lowers the comparable costs of the MMC group. Consequently, after adjustments for both case-mix and benefit differences, the MMC group is \$63 PMPM less costly than the comparison population for all members. Cost differences are largest in the 55-64 and 65 and above age groups. The MMC group is $\$ 115$ PMPM lower than the comparison population for the age category of 65 years and older, after adjustments for case-mix and benefits.

Figure 5 shows adjusted and unadjusted costs of the MMC group compared to the costs of the comparison population, by type of service. After adjusting for case-mix and benefit differences, the MMC group was less in every category, with the largest savings in professional costs (\$27 PMPM) and pharmacy (\$21 PMPM).

Figure 6 shows several Health Plan Employer Data and Information Set (HEDIS) outcomes for the population served by the MMC, the entire population served by the University of Michigan Health System, the $90^{\text {th }}$ percentile for HEDIS nationally, and the HEDIS national average. The MMC outcomes are higher than all other comparisons in every measure except "annual eye exam" for persons with diabetes, with scores that are higher than $90 \%$ of provider and payer groups reporting HEDIS measures. In addition, populations served by the MMC show better HEDIS outcomes than other populations served by the University of Michigan providers, demonstrating that the MMC provides quality enhancements above and beyond those already present in the University of Michigan Health System. 


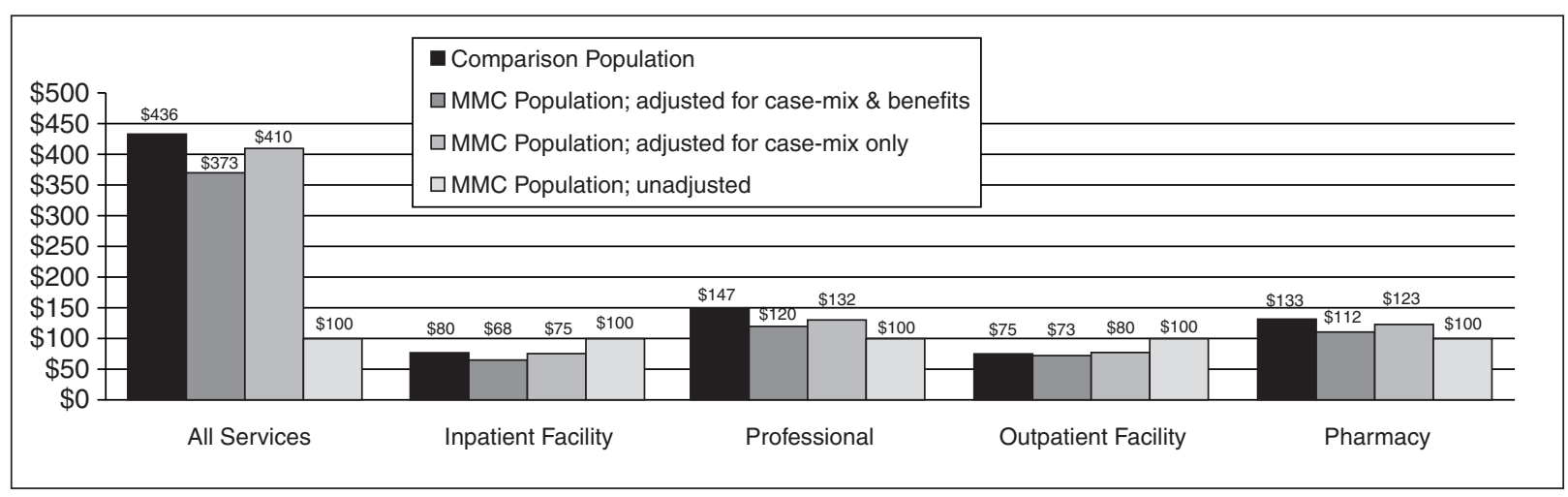

FIG. 5. Comparison of Per-Member-Per-Month (PMPM) costs by type of service.

\section{Limitations of the analysis}

To our knowledge, this study represents one of the first evaluations of coordinated medical and disease management programs to include a comparison population and appropriate adjustments for differences in fees, case-mix, and benefit design. The methods used in this evaluation allow readers to better assess the true impact of coordinated medical and disease management on utilization by removing the potential biases of extraneous factors. However, there are some limitations to the analyses reported here.

The most critical limitation of this study is the relatively small number of members in the MMC group. The ACG case-mix adjustment classifies members into clinical subsets that have relatively similar severity indices. This subclassification by the ACG system further reduces the number of members within each ACG category, thereby virtually preventing the use of a valid significance test of the cost and utilization outcomes. Consequently, even though a seemingly substantial overall difference of \$63 PMPM between the MMC group and the comparison population is reported, we are unable to assess the statistical significance of this difference.

Another limitation is that the study excluded some outpatient facility costs from the cost PMPM calculation, notably lab and emergency department costs, which can amount to $3 \%$ of the cost PMPM for a population.

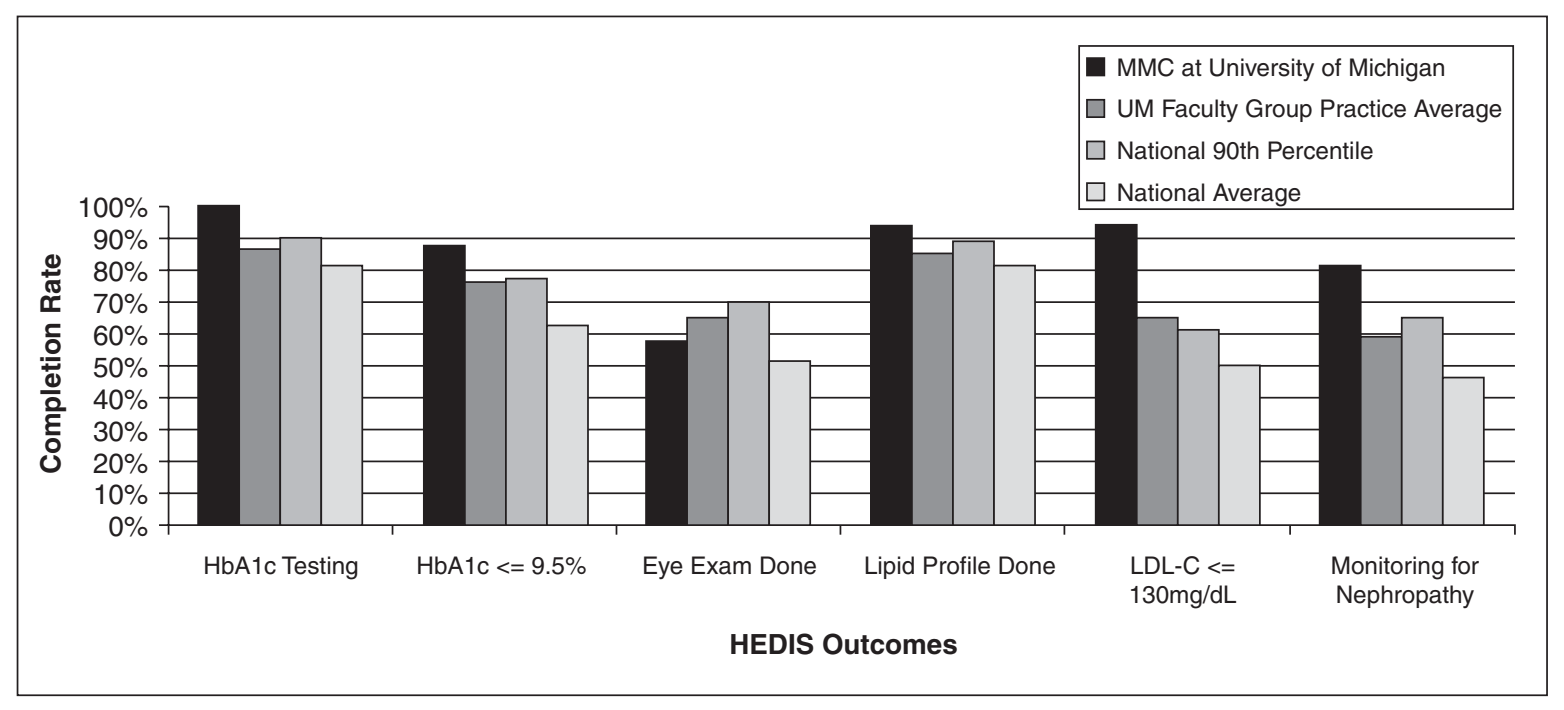

FIG. 6. Comparison of Health Plan Employer and Data Information Set (HEDIS) outcomes. 
We are also unable to isolate the specific contributions of the various components of the medical and disease management programs. For example, we cannot report the extent to which the Health Navigators reduce utilization. Instead, the study reported here is a "full body experiment" that assesses the overall impact of the medical and disease management programs as a whole.

\section{DISCUSSION}

There has been emerging literature citing the opportunities for improvements in healthcare quality and reductions in healthcare costs through integrated care coordination of complex and high-cost chronic illnesses, proactive management of chronic disease, physician leadership, and effective information exchange. The University of Michigan Health System's MMC has built the infrastructure for the application of these tools and processes, and applied these coordinated interventions to a pilot population for several years. A comparison of the cost outcomes of the population served by the MMC and those of an unmanaged population showed an overall cost reduction of \$63 PMPM. This savings represents a 2.4 to 1 return on investment (ROI). This is a conservative estimate of the ROI however, since the administrative fee for MMC services included a large amount of fixed cost (ie, data analytic support, information technology, management) that was spread across a relatively small initial volume of Partnership Health members, resulting in an inflated administrative fee of nearly \$20 PMPM. Since the time of this study, Partnership Health has more than doubled in size without any increase in the administrative fee for MMC services. Consequently, if similar cost savings are reported for the current Partnership Health enrollment, the ROI would be nearly 5 to 1 .

These findings demonstrate the potential of coordinated medical and disease management programs within a staff-model integrated delivery system that includes a robust electronic medical record and strong data analytic resources. While these components were extremely helpful in allowing program develop- ment, they are not all required. Small, private physician groups actively participated in Partnership Health as part of a broad provider network. Additionally, our later involvement with other payer demonstration projects has convinced us that these models of chronic illness management are replicable in many settings, including less cohesive or integrated systems of care. Regardless of whether it is a small or large provider group, or private practice or staff model, a key to effective systems of care coordination and chronic disease management is the commitment of clinician leaders who are integrated within the delivery system of the patient.

Small provider groups are capable of implementing many of these components. While support at the health plan level for some care management and data analytic functions may be needed, and reimbursement mechanisms for chronic illness management must be developed further, population-based medical and disease management can be implemented throughout the variety of practice models that characterize our healthcare system.

As provider groups further develop more integrated systems of medical and disease management, an important next step will be for these provider groups to learn how best to coordinate with vendor and payer-based disease management programs. All of these programs can offer positive contributions across the continuum of care. Yet it will be important for all to recognize that it is the patient's course along that continuum of care that is the focus, and the provider, payer, and vendorbased programs should coordinate with each other to achieve the optimal benefit for the patient.

\section{IN MEMORIAM}

In honor of our friend, Tom Carli. Our thoughts are with you.

\section{REFERENCES}

1. Chen A, Brown R, Archibald N, Aliotta S, Fox PD. Best practices in coordinated care. HCFA contract no. 500-95-0048 (04), 2000. 
2. Committee on Quality Health Care in America, Institute of Medicine. Crossing the quality chasm: a new health system for the $21^{\text {st }}$ century. Washington, DC: National Academy Press, 2001.

3. Wagner EH, Austin BT, VonKorff M. Improving outcomes in chronic illness. Manag Care Q 1996;4:12-25.

4. Stockwell DH, Madhavan S, Cohen H, Gibson G, Alderman $\mathrm{MH}$. The determinants of hypertension awareness, treatment, and control in an insured population. Am J Public Health 1994;84:1768-1774.

5. Kenny SJ, Smith PJ, Goldschmid MG, Newman JM, Herman WH. Survey of physician practice behaviors related to diabetes mellitus in the US: physician adherence to consensus recommendations. Diabetes Care 1993;16:1507-1510.

6. Wagner EH. Managed care and chronic illness: health services research needs. Health Serv Res 1997;32: 702-714.

7. Wagner EH, Austin BT, VonKorff M. Organizing care for patients with chronic illness. Milbank Q 1996; 74:511-544.

8. Center for the Advancement of Health. Indexed bibliography of behavioral interventions of chronic disease. Washington, DC: Center for the Advancement of Health, 1996.

9. Kinmonth AL, Woocock A, Griffin S, Spiegal N, Campbell MJ. Randomised controlled trial of patientcentred care of diabetes in general practice: impact on current wellbeing and future disease risk. BMJ 1998;317:1202-1208.

10. Bailey WC, Kohler CL, Richards JM, Jr, et al. Asthma self-management: do patient education programs always have an impact? Arch Inten Med 1999;159: 2422-2428.

11. Simon GE, VonKorff M, Rutter C, Wagner E. Randomised trial of monitoring, feedback and management of care by telephone to improve treatment of depression in primary care. BMJ 2000;320:550-554.

12. Jolly K, Bradley F, Sharp S, et al. Radomised controlled trial of follow up care in general practice of patients with myocardial infarction and angina: final results of the Southhampton heart integrated care project (SHIP). BMJ 1999;318:706-711.

13. Wells KB, Sherbourne C, Schoenbaum M, et al. Impact of disseminating quality improvement programs for depression in managed primary care: a randomized controlled trial. IAMA 2000;283:212-220.
14. Verbrugge LM, Lepkowski JM., Imanaka Y. Comorbidity and its impact on disability. Milbank Q 1989;67:450-484.

15. Boult C, Louis TA, Boult L, McCaffrey D. Chronic conditions that lead to functional limitation in the elderly. J Gerontol 1994;49:M28-M36.

16. Verbrugge L, Jette AM. The disablement process. Soc Sci Med 1993;38:1-14.

17. Blaum CC, Liang J, Liu X. The relationship of chronic disease and health status to the health services utilization of older Americans. I Am Geriatr Soc 1994;42:1087-1093.

18. Wolinsky FD, Johnson RJ. The use of health services by older adults. J Gerontol 1991;46:S345-S357.

19. Weissert WG. A new policy agenda for home care. Health Aff (Millwood) 1991;10:67-78.

20. Newcomer R, Manton K, Harrington C, Yordi C, Vertrees J. Case mix controlled service use and expenditures in the social/health maintenance organization demonstration. J Gerontol 1995;50A:M35-M44.

21. Program announcement. Model for effective chronic illness care. The Robert Wood Johnson Foundation, 2000. Available at: www.improvingchroniccare.org/ model.html. Accessed January 18, 2006.

22. Stiles RA, Mick SS, Wise CG. The logic of transaction cost economics in health care organization theory. Health Care Manage Rev 2001;26:85-92.

23. Fadel H, Odell K, Wise CG, Klinkman MS. Internal UMHS report. Care management activity in partnership health year 1. Ann Arbor, MI: UMHS, 1999.

24. Wise CG, Billi JE. A model for practice guideline adaptation and implementation: empowerment of the physician. JT Comm J Qual Improve 1995;21: 465-476.

Address reprint requests to: Christopher G. Wise, Ph.D., M.H.A. UMHS Medical Management Center University of Michigan 2500 Green Rd., Ste. 700 Ann Arbor, MI 48105-1500

E-mail: cwise@umich.edu 
This article has been cited by:

1. Cheryl Schraeder, Cynthia W Fraser, Ida Clark, Barbara Long, Paul Shelton, Valerie Waldschmidt, Christine L Kucera, William K Lanker. 2008. Evaluation of a primary care nurse case management intervention for chronically ill community dwelling older people. Journal of Clinical Nursing 17:11c, 407-417. [CrossRef]

2. Jennifer J. Hatzfeld. 2008. Developing Evidence-Based Nursing Roles: Lessons Learned From the Health Care Integrator Role. Nursing Forum 43:2, 72-79. [CrossRef]

3. Thomas Wilson, Martin MacDowell, Patricia Salber, Gary Montrose, Carolyn Hamm. 2008. Evaluation Methods in Disease Management Studies. Disease Management \& Health Outcomes 16:5, 365-373. [CrossRef] 